\title{
Simulando Mobilidades de Carga em Cristais Moleculares
}

Ingrid Gomes Ribeiro*(IC) ${ }^{1}$, Leonardo Evaristo de $\operatorname{Sousa}^{2}(\mathrm{PG})$, Pedro Henrique de Oliveira $\mathrm{Neto}^{2}(\mathrm{PQ})$ ingridgomesribeiro2@gmail.com

${ }^{1}$ Instituto de Química, Universidade de Brasília

2 Instituto de Física, Universidade de Brasília

Keywords: charge mobilities, organic electronics, Kinetic Monte Carlo.

\section{Introdução}

O estudo de transferência de carga e energia de reorganização em moléculas orgânicas é de grande relevância no campo da eletrônica orgânica, pois essas características têm grande influência sobre o funcionamento de dispositivos como OLEDs e células solares.

Neste trabalho, estudamos a energia de reorganização e a mobilidade de portadores de carga em 7 moléculas derivadas de triethylsilylethynyl. O transporte de carga é modelado pela teoria de Marcus, a partir da qual obtemos a taxa de transferência de carga. Essa taxa depende do acoplamento eletrônico e da energia de reorganização de cada molécula. Esses parâmetros são obtidos e simulações de Monte Carlo são realizadas para que as mobilidades sejam calculadas.

\section{Metodologia}

Construímos, a partir de dados cristalográficos, os cristais moleculares. Identificamos as posições nas quais o empilhamento entre anéis aromáticos é favorecido. Então, utilizando as posições preferenciais de transferência de carga previamente identificadas, variamos a distância e eventualmente a angulação entre trios de moléculas do cristal para a determinação de uma região de interação mais provável.

Calculamos a geometria de equilíbrio para as moléculas no estado neutro e carregado (positiva e negativamente). Com esses valores, calculamos a energia de reorganização de cada molécula de interesse. Em seguida, separamos dímeros nos quais são calculadas as propriedades de estrutura eletrônica e são variadas sistematicamente a distância entre esses dímeros respeitando a região de interação mais provável.

Para cada dímero calculamos o acoplamento eletrônico em todas as distâncias relativas. Utilizando uma média ponderada por uma função de Boltzmann da energia total do sistema em cada configuração da região de interação mais provável, calculamos o acoplamento eletrônico médio como função da temperatura.

Munidos dessa função de acoplamento eletrônicos e da energia de reorganização, utilizaremos a fórmula de Marcus para determinarmos a taxa de transferência de carga entre moléculas. Essas taxas juntamente com as distâncias intermoleculares serão utilizadas para o cálculo da dinâmica dos portadores de carga via um método de Monte Carlo cinético. 


\section{Resultado e Discussão}

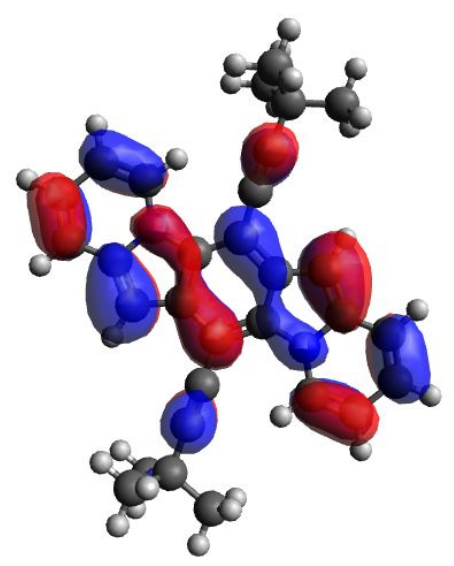

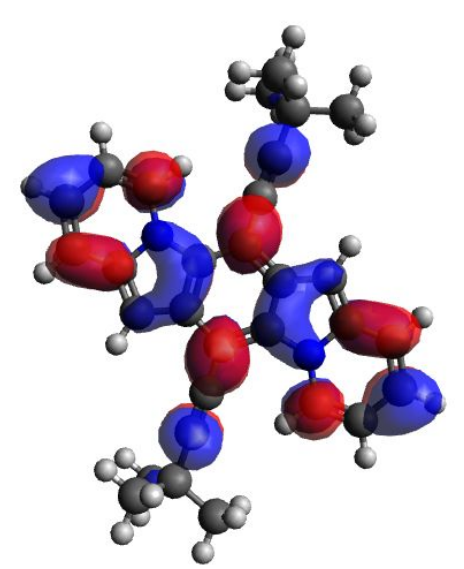

Os resultados mostram que as mobilidades obtidas por meio desse protocolo estão de acordo com resultados experimentais. O método de cálculo dos acoplamentos utilizando a média ponderada pelo fator de Boltzmann se mostra essencial para a obtenção de melhores resultados. Encontramos também a dependência da mobilidade com a temperatura para cada molécula.

\section{Conclusões}

Neste trabalho, um estudo de mobilidade de portadores de carga é feito em um conjunto de moléculas orgânicas. Energias de reorganização são calculadas usando softwares de química quântica. Da mesma forma, os acoplamentos eletrônicos também são obtidos de forma mais precisa por meio de uma média ponderada por fatores de Boltzmann. Um modelo de Monte Carlo cinético é então usado para calcular mobilidades de cargas nessas moléculas. Os resultados estão de acordo com dados experimentais e mostram o potencial dessas moléculas em aplicações em eletrônica orgânica.

\section{Agradecimentos}

Os autores agradecem o suporte financeiro da CAPES, CNPQ e da FAP-DF.

\section{Referências}

Granger, Devin B., et al. "Synthesis and Electrical Properties of Derivatives of 1, 4-bis (trialkylsilylethynyl) benzo [2, 3-b: 5, 6-b'] diindolizines." Organic letters 18.23 (2016): 6050-6053.

Günther, Florian, Sibylle Gemming, and Gotthard Seifert. "Hopping-Based Charge Transfer in Diketopyrrolopyrrole-Based Donor-Acceptor Polymers: A Theoretical Study." The Journal of Physical Chemistry C 120.18 (2016): 9581-9587. 